\title{
Exploring the Educational Effectiveness of Culturally-enhanced Serious Game in Education
}

\author{
Mazeyanti Mohd Ariffin ${ }^{1, *}$, Wan Fatimah Wan Ahmad ${ }^{1}$, and Suziah Sulaiman ${ }^{1}$ \\ ${ }^{1}$ Computer \& Information Sciences Department, 32610 Sri Iskandar, Perak, Malaysia
}

\begin{abstract}
The main objective of this study is to explore the efficiency of utilizing a culturally - enhanced serious game in education particularly in Information Technology course. To attain this objective, the academic achievement of 61 participants was measured by using quantitative QuasiExperimental research design approach. Sets of pre- and post-test questions were administered to all participants, where descriptive analysis and hypothesis evaluation were performed to the collected data. The finding revealed that there were significant increase on the mean values of participants who played culturally-enhanced serious game as compared to those who played contemporary serious game $(M=3.00)$. Therefore, the study suggested that culturally-enhanced serious game has potential to support education particularly in Information Technology course.
\end{abstract}

\section{Introduction}

Our demands, inclinations and anticipations are inspired by our own upbringing culture [1]. These demands, inclinations and anticipations determine our choice of applications or products that we use either for work, school or even at home. For example, Google simple landing page (Fig 1) is very popular in western countries. In fact, Google dominates almost $90 \%$ in most western countries and almost $80 \%$ globally [2]. However, this extremely popular search engine is struggling to grasp the attention of South Korea's users; which merely $3 \%$ of South Koreans enjoy using it. The South Koreans are more incline to use native search engine, called Naver.com (Fig 2) over Google, where Naver.com dominates $77 \%$ of the South Korean market [2]. This disproportion was influenced by culture. The issues rely not only on the language used but also on the presentation of the interface, the selection of colours and its functionalities. It was found that the western users are more incline to use a simple interface while Eastern users anticipate the search engine interface to have highly contrasting and bright colours with many hyperlinks [3, 4].

Interestingly, gamer's behaviours are also influenced by culture. Their demands and anticipation is manifested on their selection of the type of game they play as well as their behaviour during the gameplay. For example, Western gamers favour to play more challenging game type as compared to Eastern gamers [5]. Additionally, Eastern gamers prefer to avoid in-game challenges whenever they have chances (e.g. through in-app

\footnotetext{
*Corresponding author: mazeyanti2012@gmail.com
} 
purchases) as compared to Western gamers [5]. These tendencies and preferences may be attributed to the nature of Westerners' culture that enjoys doing challenging tasks and activities.

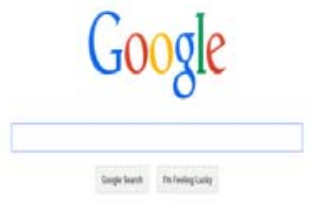

Fig. 1. Google.

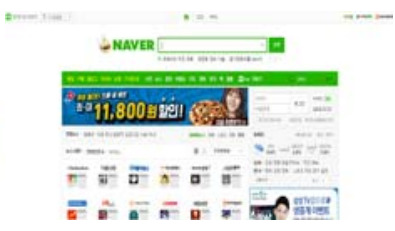

Fig. 2. Naver.com.

Furthermore, studies have revealed that culture also had influence on an individual's needs, preferences and expectations towards education [6-8]. For example, Western learners expect constructivist learning approach in contrast to Eastern learners, thus Western learners prefer to utilize social constructivist tool such as e-learning, Wikipedia and Facebook $[6,9,10]$. Additionally, the instructional expectations among learners are also vary across culture [6]. Learners from Nordic countries (e.g. Sweden, Norway and Denmark) expect constructive feedbacks as compare to learners from South Africa countries. Hence, utilizing cultural aspects in education particularly in serious game could assist to address the learner's learning needs, preferences and expectations; thus improves learner's academic performance.

The objective of this paper is to explore on the efficiency of a culturally-enhanced serious game as compared to a contemporary serious game. This paper has several parts: Part 1 is the introduction, Part 2 is the review of the literature, Part 3 is the methodology, Part 4 discusses on the findings and finally the conclusion in Part 5.

\section{Literature Review}

\subsection{Culture}

Culture can be defined as a shared pattern of mind that distinguishes the members of a group from one another [1]. These shared patterns include thoughts, manners and actions [11]. In essence, a collective or a society will have similar and distinct behaviours patterns, preferences, tendencies and expectations which differentiate them from other collective or society [6]. To describe these behavior patterns, Value Survey Model (VSM) is introduced [1]. VSM is used to distinguish one culture from another; which clarifies on the differences of a society's selections and preferences towards their own symbols, heroes, rituals and values. The VSM is extensively utilized in varies domains stretching from ICT to interpersonal communication [12,13]. This study used a serious game which was constructed based on VSM. Details of the serious game design model can be found in [4].

Current researches disclose that culture and learning are interrelated [6,14]. Evidences show that presenting learning environment that resemblance learner's culture will help to boost learners' motivation due to perceived likeliness and familiarity [9]. In spite of the connection between culture and learning, research works in this domain is still lacking particularly related to serious game $[4,6,9]$. With limited number of research works in this domain, its impact on educational effectiveness is scarce and sparse.

Though the use of VSM as a basis to generalize a culture receives critiques from some scholars, many well documented studies suggested that VSM could explain on a society's choices of symbols, preferences, behaviour, interfaces and game strategies preferences [13]. Additionally, VSM is also one of the most establish, widely replicate, has better level of 
validity as compare to other alternative models as well as repeatedly refer by scholars [12], $[13,16]$. For example, VSM was deployed in designing websites [16] while others incorporated it into user interfaces [16]. Hence, VSM is justifiable to be used in this research work in order to tackle the limited studies on culturally-enhanced serious games.

\subsection{Serious games}

Generally, computer games have story or goals, game mechanics, rules, conflict, interactivity, graphical representation and a sense of challenge or competition [17]. Meanwhile, serious games are computer games which emphasize on educational purpose instead of amusement [18]. Serious games are appropriate to be leveraged in education since they (1) provide secure and harmless environment for learners to employ their recently learnt knowledge (2) allow learners to become part of the game scenario by actively involved in the game environment and (3) motivate learners [6].

Currently, there are only a few serious games which attempt to incorporate culture aspects in its game design and game strategies. For example Smoke? [15], Trinbago Adventures of L Macawell (TALM), and Caribbean Conquest [14]. With limited number of existing culturally-enhanced computer games, its impact on educational effectiveness is scarce and limited. Thus, this paper aims to explore the effectiveness of a constructed culturally-enhanced serious game (called my-CEN) over a contemporary serious game (called CyberCIEGE).

\section{Methodology}

\subsection{Research design}

This study employed Quasi-Experimental research design approach. 61 participants were involved in this study and they were randomly divided into two groups; experimental 1 (X1) and experimental 2 (X2). Participants in X1 were required to play CyberCIEGE while participants in $\mathrm{X} 2$ were required to play my-CEN. All participants were also required to take pre- and post-test in order to assess their academic performance. The pre- and post-test questions have 3 parts: Part A is the demography, Part B is about serious game experience and finally Part $\mathrm{C}$ is formulated to assess the participants' knowledge in computer security principles and malicious threats. The academic performance scores are analysed by using two approaches; descriptive analysis and hypothesis evaluation. A null hypothesis $\left(\mathrm{H}_{0} 1\right)$ was created and stated as below:

$H_{0} 1$ : There is no significant difference on acquisition of knowledge between X1 and X2 group.

To assess the academic performance of each participant, the pre-test scores were subtracted from the post-test scores and Independent Samples t-Test using $\alpha=0.05$ was performed to test $\mathrm{H}_{0} 1$.

\subsection{CyberCIEGE serious game}

CyberCIEGE is a serious game developed by Rivermind Inc. with collaborators from academicians and developers from United States [19]. CyberCIEGE serious game is a realtime strategy (RTS) serious game that requires player to administer computer systems of a company. Player is given an initial budget to buy setup and sustain the computer systems 
from malicious code attack. Player must balance the computer security level and the employee's productivity within the given budget and time frame. Computer security implementation which is extremely strict ensures $100 \%$ security of the computer systems however will make workers incapable to conduct their task, which indirectly contribute to the reduction of the company's productivity.

\section{3 my-CEN serious game}

My-CEN was customized and enhanced from CyberCIEGE. It was customized and extended based on author's game design model called GADEM. Generally, GADEM is a model which facilitates game designers and game developers to develop a culturallyenhanced serious game. GADEM was grounded on Intercultural Communication and Social Construction of Technology theories. Detail information on GADEM can be found in [4]. My's-CEN game environment was developed to be similar to the learner's culture which in this case is Malaysian culture. Therefore, my-CEN is considered as a culturally-enhanced computer game.

\section{Results and discussion}

61 first year students from Computer \& Information Sciences Dept. in Universiti Teknologi PETRONAS participated in this study. 30 participants were randomly assigned to X1 while the remaining 31 participants were assigned to X2. The number of participants participate in this work was justified since according to ref [20], the sample size from 30 to 500 was sufficient to produce significant result.

$67.00 \%$ of participants in X1 are male while only $20.00 \%$ of participants in X2 are male. On average, participants in X1 spent approximately 9.33 hours per week playing computer games while participants in X2 spent about 9.45 hours per week for the same activity. This result suggested that both groups had quite similar time spent and computer game exposure.

Additionally, the result also revealed that "Strategy" and "Role-Playing" game were the most popular game genre played by the participants regardless of gender. Meanwhile, "Racing" and "Dance" were the least popular game genre. These patterns were demonstrated in both groups. Since majority of the participants preferred to play "Strategy" game genre, hence it could be inferred that these participants were accustomed with game interfaces components, navigation scheme and input mappings of strategy games.

\subsection{Descriptive analysis result}

The descriptive analysis result of total increment scores for each group is depicted in Figure 3. About $65 \%$ of participants in X1 showed improvement in their post-test while an astounding of $90 \%$ of participants in X2 improved their scores on the same test. Hence, it clearly showed that both groups performed better in their post-test as compared to their pretest regardless the type of serious game that they played.

Figure 4 provides more detail evidence on the performance of the participants of both groups. Majority of participants in X1 showed low improvement in between 1 to 5 marks (78.9\%) as compared to only $44.4 \%$ in X2. Another $15.8 \%$ of participants in X1 improved moderately in between 6 to 10 marks while $48.1 \%$ of participants in X2 improved moderately. Finally, $7.4 \%$ of participants in X2 demonstrated sheer improvement in their post-test as compared to only $5.3 \%$ in X1. Although both groups demonstrated positive improvement in their post-test, however participants in X2 scored higher in terms of marks distributions. Almost half (49\%) of participants in X2 improved their scores in between 6 to 
10 marks as compared to participants in X1. Additionally, more participants in X2 obtained more than 10 marks as compared to participants in X1. Hence, it could be inferred that participants in X2 demonstrated better achievement as compared to participants in X1.

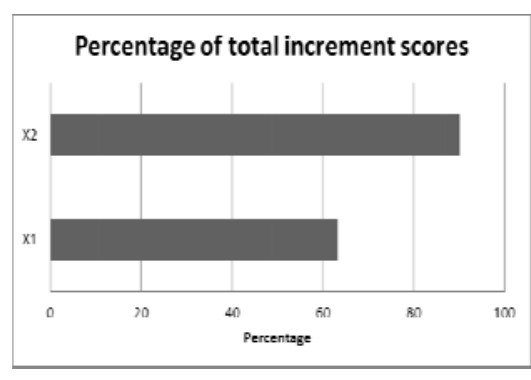

Fig. 3. Percentage of total increment scores.

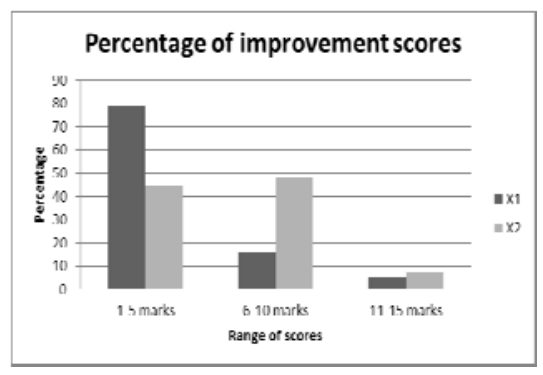

Fig. 4. Percentage of improvement scores.

\subsection{Hypothesis evaluation}

In order to provide more significant evidence, an Independent Samples t-test was conducted to compare the total increment scores for X1 and X2. Table 1 shows the Independent Samples t-test result of $\mathrm{H}_{0} 1$.

Table 1. Independent Samples t-Test result for $\mathrm{H}_{0} 1$

\begin{tabular}{|c|c|c|c|c|}
\hline \multicolumn{5}{|c|}{ Group statistics } \\
\hline & Group & $\mathrm{N}$ & Mean & Std. Deviation \\
\hline Marks & $\mathrm{X} 1$ & 30 & 2.0667 & 3.66656 \\
\hline & $\mathrm{X} 2$ & 31 & 5.0000 & 3.26599 \\
\hline
\end{tabular}

\begin{tabular}{|c|c|c|c|c|}
\hline \multicolumn{5}{|c|}{ Independent Samples Test } \\
\hline & $\mathrm{t}$ & $\frac{\mathrm{df}}{\mathrm{m}}$ & $\frac{\text { Sig. (2-tailed) }}{\mathrm{m}}$ & Mean Difference \\
\hline Marks & -3.296 & 57.730 & .002 & .2 .93333 \\
\hline
\end{tabular}

From Table 1, it showed that the mean value of $\mathrm{X} 2$ is higher $(\mathrm{M}=5.00)$ as compared to mean value of $\mathrm{X} 1(\mathrm{M}=2.00)$. Additionally, Independent Samples t-test showed that there is significant difference in scores for $\mathrm{X} 1(\mathrm{M}=2.00, \mathrm{SD}=3.66)$ and $\mathrm{X} 2(\mathrm{M}=5.00, \mathrm{SD}=3.26)$; $\mathrm{t}$ $(59)=3.296, p=0.002$ (two-tailed). This finding suggests that the null hypothesis is rejected. Therefore, this result indicates that participants in X2 demonstrated higher academic achievement as compared to the participants in X1.

\section{Conclusion}

The findings from both descriptive analysis and hypothesis evaluation suggested that $\mathrm{H}_{0} 1$ was substantiated. It showed that there is significant improvement in terms of academic performance between participants playing culturally-enhanced serious game and participants who played a contemporary serious game. Hence, it could be concluded that participants in X2 performed better as compared to participants from X1. This finding suggests that learning from environment that is culturally similar to the learners may influence the performance of the learners due to familiar and similar cultural context. This is consistent with recommendation made by scholars in Intercultural Communication who highlighted on the deniable connection between learning and culture. Hence, this study concludes that culturally-enhanced serious game has potential to increase student's academic achievement as compared to learning by using contemporary serious game. 


\section{References}

1. G. Hofstede and G. Hofstede, Cultures and organizations: Software of the mind. (MacGraw Hill, 2005).

2. K. Reinecke, "Culturally Adaptive User Interfaces," Unpublished $\mathrm{PhD}$ thesis, University of Zurich, Germany, (2010).

3. K. Reinecke and A. Bernstein. ACM Trans. Comput. Interact, 18, 2 (2011).

4. M. A. Mazeyanti, W. A. Wan Fatimah, and S. Suziah. J. Eng. Appl. Sci., 10, 23 (2015).

5. InMobi, "Mobile Gaming Cross-Market Analysis: First Edition, 2014 Q1,” (2014).

6. M. A. Mazeyanti, W. A. Wan Fatimah, and S. Suziah, "Culturally Enhanced Computer Game: The Impact on Educational Effectiveness," .Mediterranian J. Soc. Sci., vol. in press, 2015.

7. R. C. S. Yee, "Perception of Online Learning in an Australian University: Malaysian Student's Perspectives," Queensland University of Technology, (2011).

8. D. Subramony, "Socio-Cultural Issues in Educational Technology Integration," Build. Blocks Soc. Found, 6, 1, (2011).

9. P. Mohammed and P. Mohan, Caribb. Teach. Sch., 1, 1 (2011).

10. W. Littlewood. Lang. Teach. Res., 5, 1 (2001).

11. Jupit, Amelia Jati Robert, et al. "Cross-cultural awareness in game-based learning using a TPACK approach." The 10th International Workshop on Internationalisation of Products and Systems.(11-14 July 2011). (2011).

12. I. Zurida, J. Azizah, and A. Azlina. Procedia - Soc. Behav. Sci., vol. 65. (2012).

13. K. Reinecke and A. Bernstein. MIS Q., 37, 2 (2013).

14. P. Mohammed and P. Mohan, "Combining Digital Games with Culture: A Novel Approach towards Boosting Student Interest and Skill Development in Computer Science Programming," in Proceedings of International Conference on Mobile, Hybrid, and On-Line Learning, (2010).

15. R. Khaled, P. Barr, R. Biddle, R. Fischer, and J. Noble, "Game design strategies for collectivist persuasion," in Proceedings of ACM SIGGRAPH Symposium on Video Games - Sandbox, (2009).

16. B. Duygu, "Cultural Factors in Web Design," J. Theor. Appl. Inf. Technol., 3, 7 (2009).

17. A. Derryberry, "Serious games : online games for learning." (Adobe Ltd, 2007).

18. Ariffin, Mazeyanti Mohd, Wan Fatimah Wan Ahmad, and Suziah Sulaiman. "Guideline for Designing an Effective Serious Game by Using Cultural-Based Game Design Model." International Visual Informatics Conference. Springer International Publishing, (2015).

19. M. Thompson and C. Irvine, "Active Learning with the CyberCIEGE Video Game," in Workshop on Cyber Security Experimentation and Test, (2011)

20. U. Sekaran, Research method of business: A skill-building approach, (Fourth Edi. John Wiley \& Sons, 2006). 